\title{
Tacrine alleviates neuropathic pain in mice by mimicking the cell adhesion molecule L1
}

Hua-Li Xu ${ }^{1}$, Shi-Yuan $\mathrm{Xu}^{1, *}$

${ }^{1}$ Department of Anaesthesiology, Zhujiang Hospital of Southern Medical University, 510282 Guangzhou City, Guangdong, China

\section{*Correspondence}

xsy998@smu.edu.cn

(Shi-Yuan Xu)

\begin{abstract}
Neuropathic pain, which is caused by nervous system damage or dysfunctions, remains one of the most intractable challenges in modern medicine due to the lack of effective drugs. Tacrine, which is a small organic compound, is known to mimic the beneficial characteristics of the neural cell adhesion molecule L1 (L1CAM, L1) in vitro. Although previous studies indicated that $\mathrm{L} 1$ constitutes a viable strategy for promoting regeneration after nervous system injury, it is not clear whether L1 has a definite role in peripheral nerve injury. In this study, we observed that tacrine eased thermal hyperalgesia and mechanical allodynia after sciatic nerve chronic construction injury and restored functional morphological damage. Furthermore, tacrine suppressed the proliferation and activation of glia and reduced the level of IL-1 $\beta$, IL- 6 and TNF$\alpha$. Tacrine also inhibited the JAK2/STAT3 signaling pathway, which is involved in neuroinflammation. These observations indicated that tacrine is a promising candidate for an analgesic agent for neuropathic pain.
\end{abstract}

\section{Keywords}

Neuropathic pain; L1; Tacrine; Neuroinflammation; JAK2/STAT3 pathway

\section{Introduction}

Neuropathic pain (NP), which is caused by lesions or disease of the somatosensory nervous system, is a common type of chronic pain affecting 7-8\% of the global population [1]. However, the curative effect of the available therapies for clinical neuropathic pain remains poor because of the complex causes and complicated mechanisms behind such pain. There is therefore an urgent need to find novel therapeutic approaches to ease neuropathic pain in patients. The clinical manifestation of NP is hyperalgesia and allodynia (amplified responsiveness to painful stimuli, and a painful response to normally non-noxious stimuli, respectively) [2]. Overactivity in pain-sensing neurons induces secondary hyperexcitability in neurons upstream of the ipsilateral spinal dorsal horn. A substantial amount of research has confirmed that the proliferation of glial cells and the interaction of neural networks play a key role in the initiation and maintenance of NP [3, 4]. In the course of neuro inflammation, noxious cytokines activate the Janus kinase 2 (JAK2) signal transducer and activator of transcription 3 (STAT3) signal pathway, which is involved with central sensitization [5]. Therefore, the suppression of neuro-inflammatory cascades is considered to be a potential therapeutic strategy [6]. Given the heterogeneous nature of the processes that support the development of neuropathic pain, the underlying molecular mechanisms can be better elucidated using lesion models.

Cell adhesion molecule L1 (L1CAM, L1), which is a mem- ber of the immunoglobulin superfamily, is widely expressed in neurons [7-9]. Moreover, L1 has been shown to be a potential beneficial molecule for promoting not only axonal fasciculation and regeneration, but also for increasing neuronal survival, and promoting remyelination and synaptic plasticity in a repressive environment [10-13]. L1 mediates cell-cell adhesion in intracellar signaling cascades by binding partners $[14,15]$ such as Mitogen-activated protein kinase (MAPK) systems and fibroblast growth factor receptor $1[16,17]$. L1 has been regarded as a promising molecule for treating the diseased nerve system. However, it is not yet clear whether L1 is effective in the NP mouse model. The viral delivery of L1 and the injection of stem cells over-expressing L1 would present difficulties if translated to human clinical therapy [18]. Conventional analgesic agents such as nonsteroidal anti-inflammatory drugs, opioids, and anti-depressant drugs inevitably exhibit adverse effects that limit their application in treatment of clinical neuropathic pain $[19,20]$. Therefore, FDA-approved small molecule mimics could be more acceptable because of their pharmacokinetics and accessibility.

Tacrine (1, 2, 3, 4-tetrahydroacridine-9 hydro chloride; Bristol, UK) is a reversible acetylcholinesterase inhibitor with pharmacological action on ion channels and monoamine levels. It is a small organic molecule and has been shown to mimic the useful features of L1. Tacrine has been used for treating memory loss and cognitive decline in Alzheimer's dementia $[21,22]$, and was recently verified as promoting functional recovery, neurite outgrowth, and neuronal survival in zebrafish 
after spinal cord injury [23]. Because of its resemblance to the extracellular region of L1, Tacrine stimulates L1 expression and proteolysis, especially the phosphorylation of kinases of neurons in vitro. Nevertheless, the effect of Tacrine on pain is unknown. Therefore, our study was designed to use an experimental model of neuropathic pain to investigate any analgesic effect of Tacrine, and to elucidate any underlying mechanism behind its action.

\section{Materials and methods}

\subsection{Animals}

Male C57BL/6 mice (4-6 weeks old) weighing 20-25 g were obtained from the Animal Experimental Center of Guangdong, China (Laboratory Animal Certificate: NO: 44200300015927). The mice were housed at the animal center of Guangdong-Hongkong-Macau Institute of CNS Regeneration (GHMICR) of Jinan University, under standard conditions of humidity and temperature, a $12 \mathrm{~h}$ of light/dark cycle, and free access to food and water. Sixty mice were allocated to 3 groups ( $n=20$ /group) as follows: (1) Sham Group; (2) Surgery + Vehicle Group; (3) Surgery + Tacrine Group. Drugs were administered after surgery.

\subsection{Mouse Chronic Constriction Injury (CCI) model and drug administration}

Mice were anesthetized with $10 \%$ chloral hydrate. Then the left sciatic nerve was exposed through a small incision. Unilateral sciatic nerve constriction was performed with three loose ligatures using 5-0 silk. Ligatures were spaced at 1$\mathrm{mm}$ intervals. In the Sham Group, the sciatic nerve was just exposed but not ligatured.

Intrathecal injections were performed using a $10 \mu \mathrm{L}$ microinjection syringe. The needle was inserted into the intervertebral space of the L5-6 region of the spinal cord and 10 $\mu \mathrm{L}$ of solution was injected. The reflexive flick of the tail indicated a successful injection. The Sham group received 10 $\mu \mathrm{L}$ of normal saline

Tacrine was freshly diluted with normal saline and was injected intrathecally into the L5-6 regions at a dose per mouse of $0.2 \mathrm{mg} / \mathrm{mL}$ [24] for 28 days after surgery.

\subsection{Behavioral testing}

The mice underwent daily habituation for 2 days before baseline testing. The mice were placed in an individual Plexiglas chamber with an elevated mesh screen for $30 \mathrm{~min}$ before testing. All behavioral tests were carried out $1 \mathrm{~h}$ intervals.

Paw-withdrawal frequencies (PWFs) were tested using von Frey fibers. The calibrated von Frey filaments of $0.4 \mathrm{~g}$ were used to stimulate the hind paw for $1 \mathrm{~s}$ and repeated 10 times. Then PWF was represented as a \% response frequency ([number of paw withdrawals $/ 10$ trials] $\times 100=\%$ response frequency).

Paw withdrawal latencies (PWTLs) to noxious heat stimulation were tested with the Analgesia Meter (IITC Inc. Life Science Instruments). In brief, each mouse was placed in an individual Plexiglas chamber on a glass plate. A beam of light passed through a hole in the chamber and stimulated the middle of each hind paw. If the mouse withdraws its foot, the light beam automatically turns off. The PWTL was recorded as the length of time from the start of light beam to the foot withdrawal, five times, with a cut-off time of $20 \mathrm{~s}$ being applied to avoid hind paw injury. Cold hyperalgesia was assessed using the cold plate test in mice [25]. Each mouse was placed onto the cold plate in turn (IITC Inc. Life Science Instruments) at a temperature of $5^{\circ} \mathrm{C} \pm 0.2^{\circ} \mathrm{C}$. The frequency of lifting, flinching, or scratching its hind paw was recorded. Each ipsilateral and contralateral hind paw was tested for 5 $\min$.

\subsection{Immunofluorescence staining}

Mice were perfused with $200 \mathrm{~mL}$ of $4 \%$ paraformaldehyde (pH 7.4) after being anesthetized with $3 \%$ isoflurane. L5 segments were then harvested, fixed at $4{ }^{\circ} \mathrm{C}$ for at least $4 \mathrm{~h}$ and then dehydrated in $30 \%$ sucrose overnight. The tissues were sectioned at a thickness of $20 \mu \mathrm{m}$ on a freezing microtome. The sections were then blocked with PBS containing 10\% goat serum and $0.3 \%$ Triton $\mathrm{X}-100$ for $2 \mathrm{~h}$ at $37{ }^{\circ} \mathrm{C}$, and then incubated at $4{ }^{\circ} \mathrm{C}$ with primary antibody overnight. They were then incubated for $2 \mathrm{~h}$ at room temperature with the second antibody. All immunofluorescence images were observed using a Leica DMI4000 fluorescence microscope and captured with a DFC365FX camera (Leica, Germany).

\subsection{Western blot analysis}

After administration of tacrine for 28 days, mice were decapitated and the ipsilateral L5 spinal cords were removed. The tissues were separately homogenized with ice-cold lysis buffer containing $0.25 \mathrm{M}$ sucrose, $10 \mathrm{mM}$ Tris, $2 \mathrm{mM} \mathrm{MgCl} 2,5 \mathrm{mM}$ EGTA, $1 \mathrm{mM}$ phenyl methylsufonyl fluoride, $1 \mathrm{mM}$ DTT, and $40 \mathrm{mM}$ leupeptin. After the crude homogenate was centrifuged at $4{ }^{\circ} \mathrm{C}$ for $15 \mathrm{~min}$ the supernatants were collected and the total protein concentration in each sample was measured by BCA protein assay (Keygen Biotech, China). Each sample was heated at $99{ }^{\circ} \mathrm{C}$ for $10 \mathrm{~min}$ and then loaded onto a $4 \%$ stacking/10\% separating SDS-polyacrylamide gel (Bio-Rad Laboratories). Then the protein was transferred onto a PVDF membrane (Millipore, USA). After blocking of nonspecific binding sites by $5 \%$ non-fat milk in Tris-buffered saline containing $0.1 \%$ Tween-20 for $1 \mathrm{~h}$, the membranes were incubated with the respective primary antibody overnight. These antibodies included mouse anti-L1 (1 : 1000, ab24345, Abcam), rabbit anti-JAK2 (1 : 2000, ab108596, Abcam), anti-STAT3 (1 : 1000, ab68153, Abcam), and mouse $\beta$-actin ( $1: 2000,3700$ s, CST), overnight at $4{ }^{\circ} \mathrm{C}$. The membranes were washed three times for $10 \mathrm{~min}$ each, and then incubated with horseradish peroxidase conjugated IgG anti-mouse (1:3000, 7076s, CST) or IgG anti-rabbit secondary antibody $(1: 3000,7074 \mathrm{~s}, \mathrm{CST})$, and were visualized by western peroxide reagent and luminol/enhancer reagent (Clarity western ECL Substrate, BioRad). Image lab software was used to exposed and quantified the intensity of the membranes. 
A
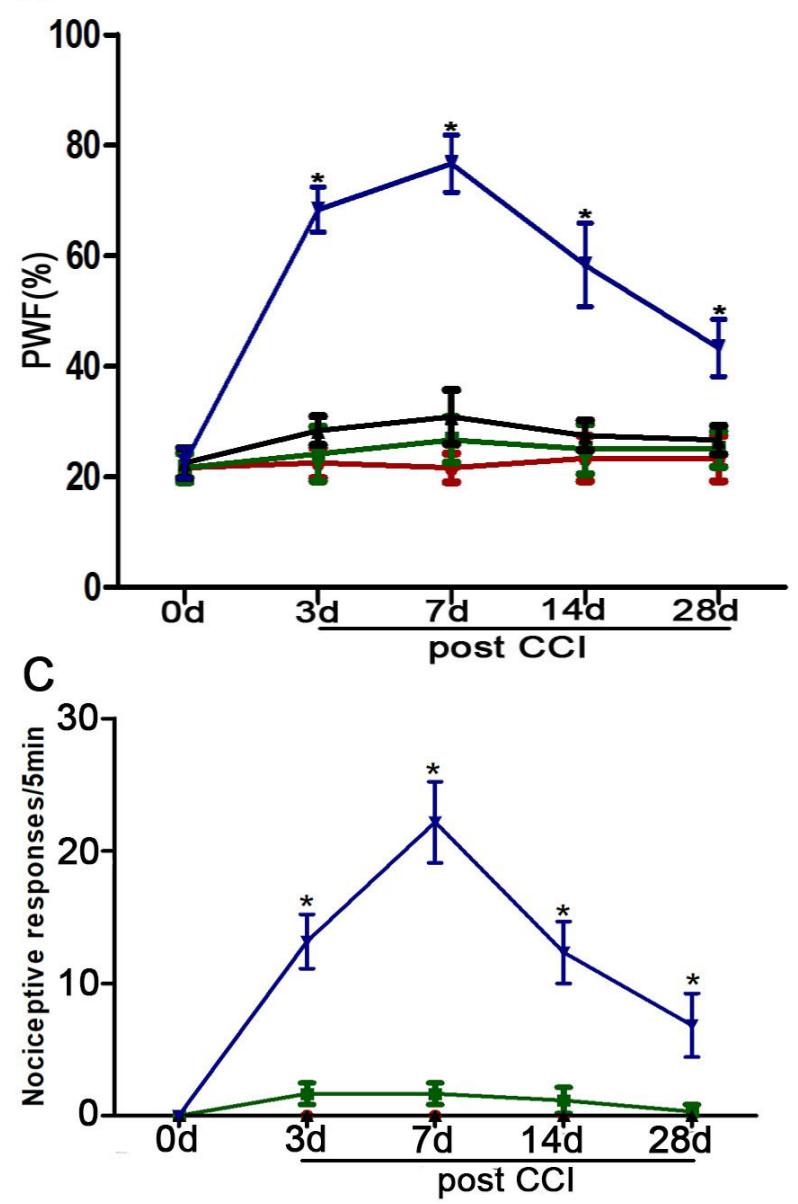

B

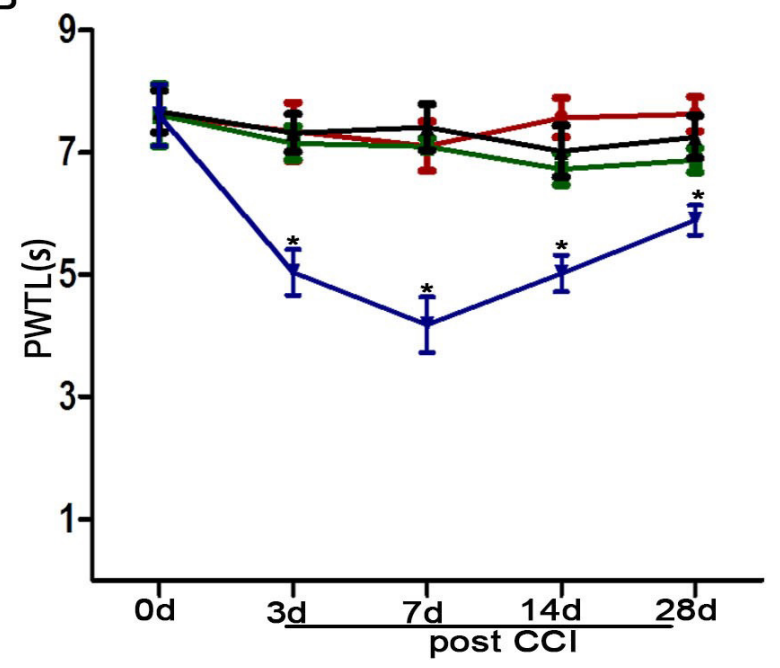

- ipsilateral sham
$\rightarrow$ contralateral sham
$\rightarrow$ ipsilateral CCI
$\rightarrow$ contralateral $\mathrm{CCl}$

FIGU RE 1. CCI induced mechanical allodynia, heat hyperalgesia and cold allodynia throughout the experimental period of 28 days. Data are expressed as mean $\pm \mathrm{SEM} ; \mathrm{n}=10$ per group. (A) The bilateral paw withdrawal frequencies (PWFs) to mechanical stimuli were evaluated in the sham and CCI groups $\left({ }^{*} p<0.05\right.$ versus the sham ipsilateral group). (B) The bilateral paw withdrawal latencies (PWTLs) were detected in each group $\left({ }^{*} p<0.05\right.$ versus the sham ipsilateral group). (C) The change of nociceptive response time in cold plate test in each group $\left({ }^{*} p<0.05\right.$ versus the sham ipsilateral group).
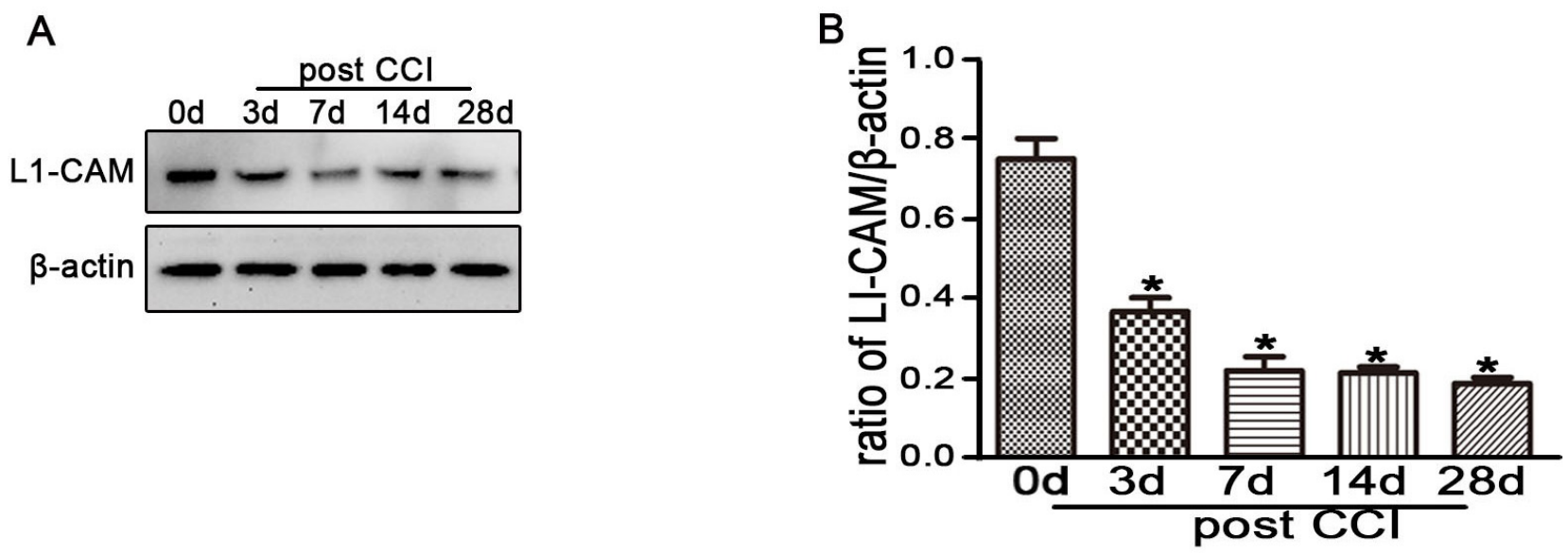

F I G U RE 2. L1 expression was decreased in the dorsal spinal cord during CCI-induced NP. (A) L1 expression in spinal cord detected by western blotting after CCI. (B) Statistical analysis of the differences between groups $(\mathrm{n}=3)$. Data are presented as the means \pm SEM. $* p<0.05$ versus the sham group.

\subsection{Enzyme-linked immunosorbent assay (ELISA)}

The protein level of interleukin-1 $\beta$ (IL-1 $\beta$ ), interleukin-6 (IL$6)$ and tumor necrosis factor- $\alpha(\mathrm{TNF}-\alpha)$ were measured by
ELISA kits (R\&D Systems, Minneapolis, MN, USA) using the manufacturers' protocol. 
A<smiles>Nc1c2c(nc3ccccc13)CCCC2</smiles>

C

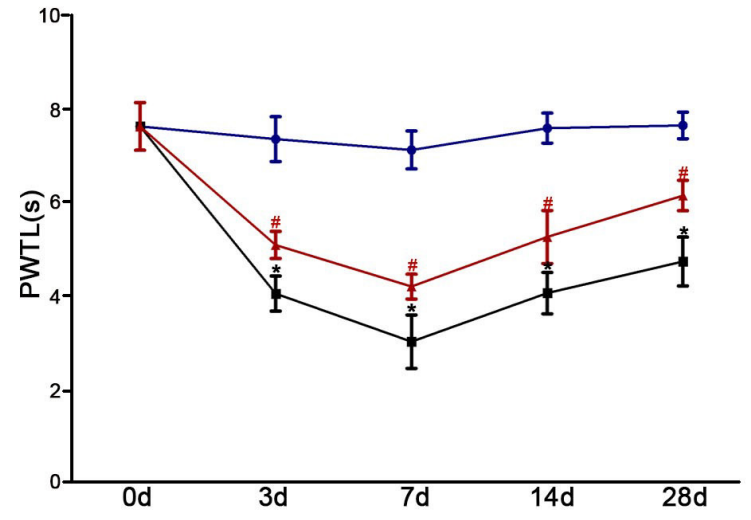

B

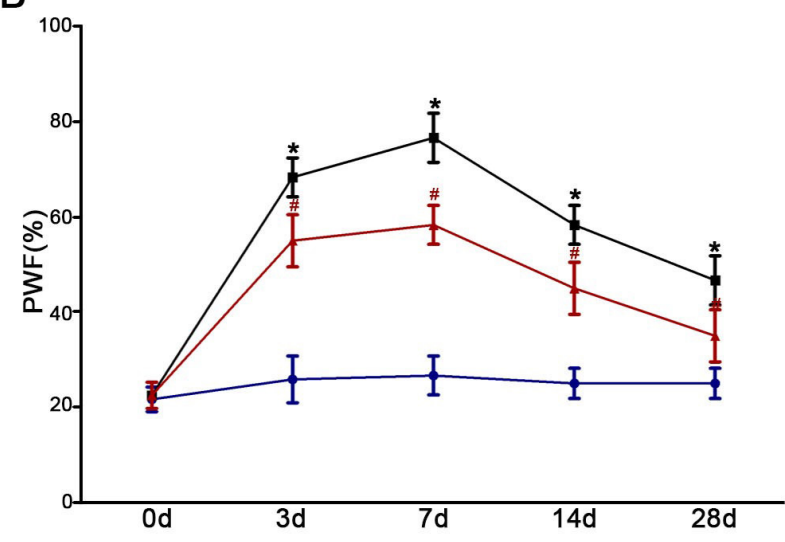

D

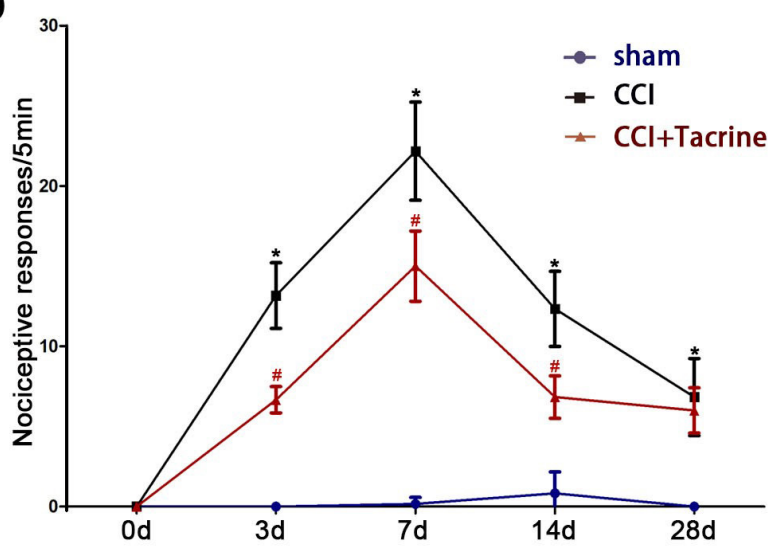

F I G U R E 3. Tacrine treatment prevents CCI-evoked hyperalgesia in mice. (A) The chemical structural formula of tacrine. (B) The ipsilateral paw-withdrawal frequency (PWF) to mechanical stimuli in each group $(\mathrm{n}=10)$. (C) The ipsilateral pawwithdrawal latency (PWTL) to heat stimuli of different groups $(n=10)$. (D) The ipsilateral paw nociceptive responses to cold plate in each group $(\mathrm{n}=10)$.

\subsection{Statistical analysis}

Statistical calculations and analyses were carried out using GraphPad 6.0 (GraphPad Software, La Jolla, CA, USA). Data were presented as the mean \pm SEM. The difference between two groups was analyzed by a two-tailed Student's test. Then $5 \%$ or less confidence interval was considered to be statistically significant.

\section{Results}

\subsection{CCI-induced mechanical allodynia, heat hyperalgesia and cold allodynia over time}

The CCI model was established in mice to simulate neuropathic pain. Compared with sham-operated groups and the contralateral hind paw of the CCI group, the PWF of the ipsilateral CCI group significantly increased and remained at a high level for 4 weeks ( $p<0.05$, Fig. 1A). CCI also induced a significant heat hyperalgesia in mice. The PTWL of the ipsilateral hind paw decreased, showing increased sensitivity to radiant heat ( $p<0.05$, Fig. 1B). Fig. $1 \mathrm{C}$ showed the coldallodynia responses of each group. After CCI, the mean time of response showed a peak on day 7 and then decreased gradually.

\subsection{CCI reduced the expression of L1 protein in spinal cord}

To explore L1 during NP, we examined the expression of protein in L5 spinal cord by western blotting (Fig. 2A). The level of L1 decreased over time after CCI surgery compared with the sham group ( $p<0.05$, Fig. $2 \mathrm{~B})$, indicating that L1 may be involved in the pathological process of $\mathrm{CCI}$-induced NP.

\subsection{Intrathecal injection of Tacrine alleviates CCI-induced pain hypersensitivity}

We tested the effect of Tacrine on alleviating pain and inflammation caused by CCI through behavioral test. The chemical structure of Tacrine was shown in Fig. 3A. There was no variation in PWF between groups before surgery (Fig. 3B). Mice subjected to CCI injury showed persistent mechanical allodynia compared to the sham group $(p<0.05)$. However, administration of Tacrine resulted in an obvious decrease in PWF in comparation with the CCI vehicle group $(p<0.05)$. Data showing the effects of Tacrine on PWTL is presented in Fig. 3C. There was no significant between-group difference $(p>0.05)$ in PWTL before CCI surgery. Compared with the sham group, the CCI mice were obviously more sensitive 
A
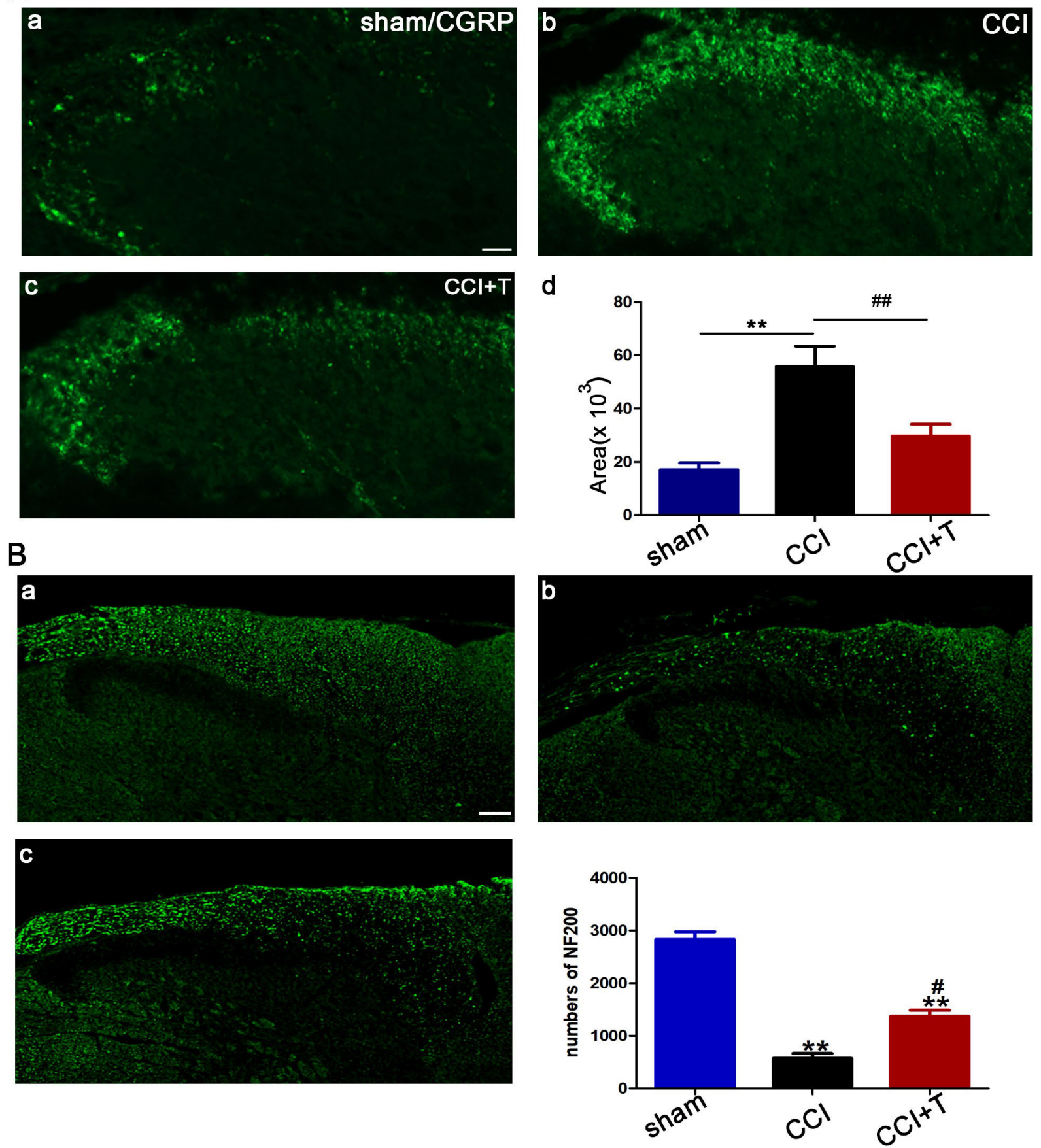

F I G U R E 4. Tacrine changed the expression of CGRP and NF200 after CCI-injury. (A) Anti-CGRP immunostaining was carried out transverse spinal sections in sham group (A-a), CCI group (A-b) and CCI + Tacrine group (A-c). Statistical analysis of integrated density of anti-CGRP fiber in each group $(d, n=6)$. (B) Anti-NF200 immunostaining of transverse spinal sections in sham group (B-a), CCI group (B-b) and CCI + Tacrine group (B-c). Statistical analysis of numbers of NF200 in each group (n =6). ${ }^{*} p<0.05,{ }^{* *} p<0.01$ versus the sham group; ${ }^{\#} p<0.05,{ }^{\# \#} p<0.05$ versus the CCI group. Scale bars indicated $200 \mu \mathrm{m}$.

to the radiant heat over 28 days after injury. However, the mice receiving Tacrine exhibited a significantly longer effect than did the CCI group ( $p<0.05$ ) (Fig. 3C). Different groups of mice were also given the cold plate test (Fig. 3D). The mice did not show any significant between-group difference in the baseline data. After CCI, the vehicle group number of responses was markedly increased, especially on Day 7 post surgery, whereas, the Tacrine group $(250 \mathrm{nmol} / \mathrm{d})$ showed significantly fewer responses $(p<0.05)$ (Fig. 3D). From the behavioral results above, it seems clear that Tacrine alleviated CCI-induced pain hypersensitivity.

\subsection{Tacrine changed the expression of CGRP and NF200 in spinal cord}

We studied nociceptive fibers by using anti-calcitonin, generelated peptide (CGRP) immunostaining. As shown in Fig. 4A, a,b,c, CGRP-positive fibers mostly terminated in lamina I of spinal dorsal horn. The positive expression of CGRP was 

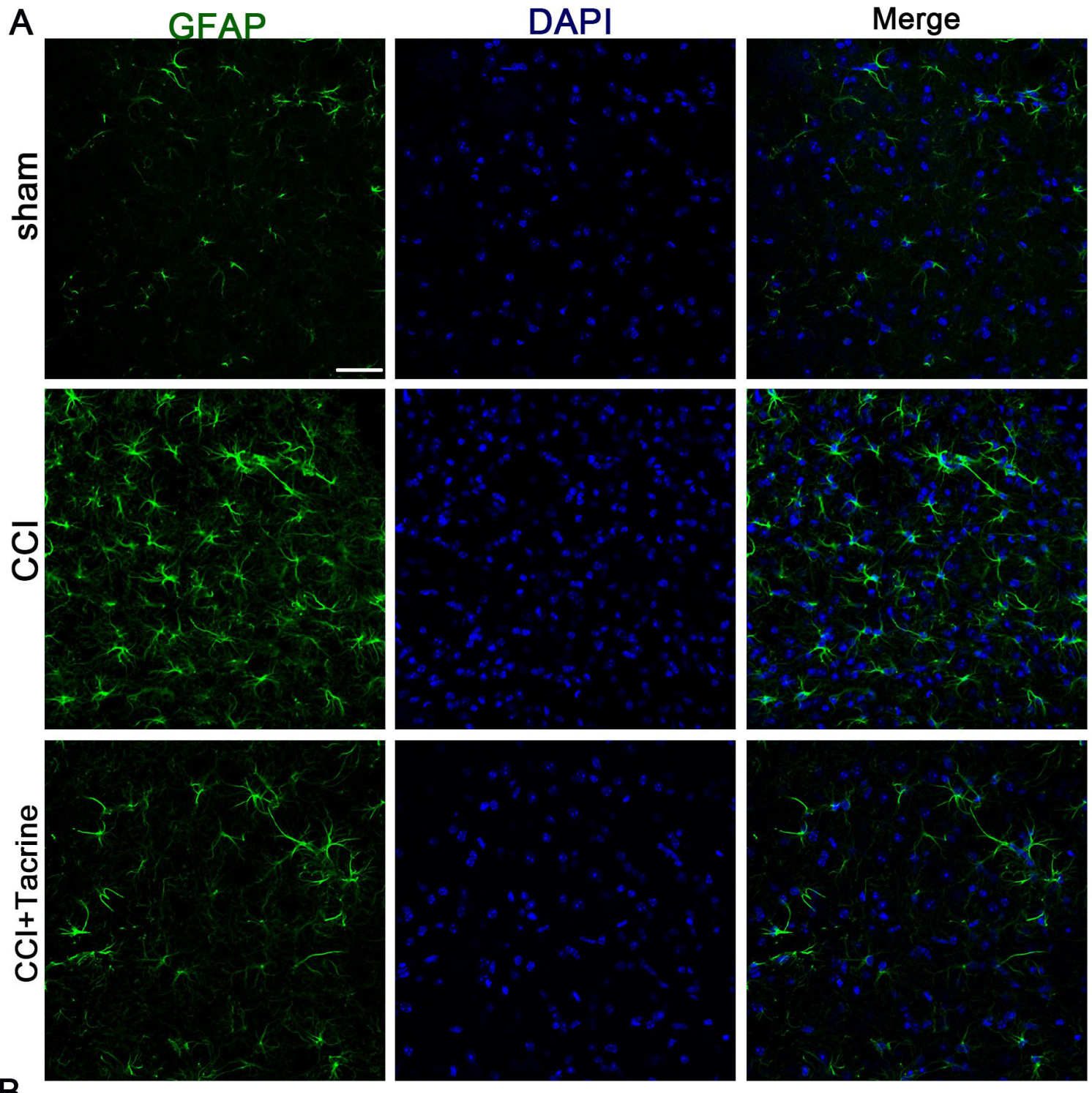

B
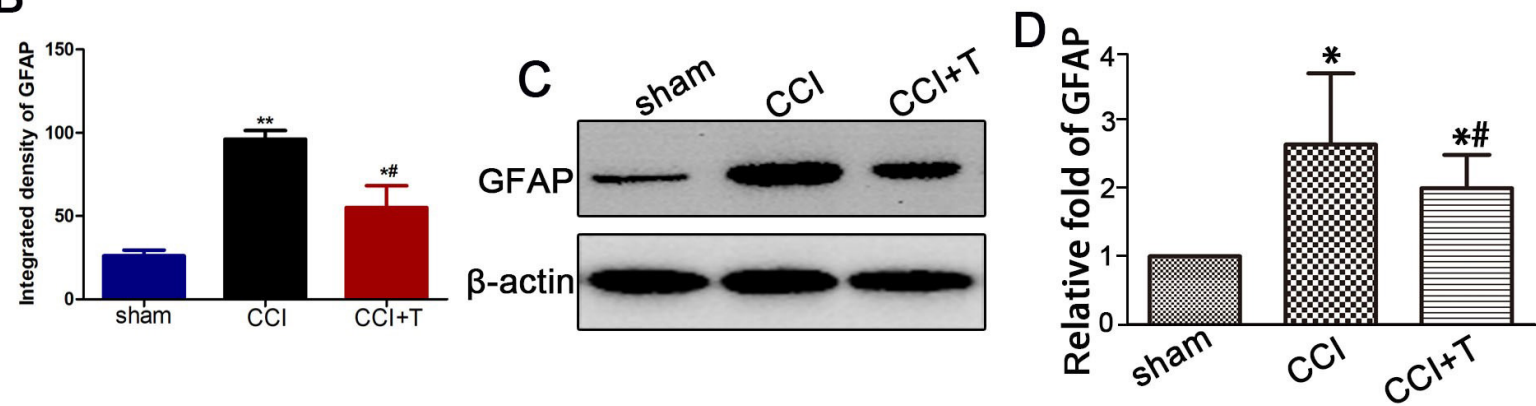

F I G U RE 5. Tacrine depressed the activation of astrocytes after CCI-injury. (A) Representative immunofluorescence staining of the astrocytic marker GFAP and the nucleus marker DAPI on the ipsilateral spinal cord (scale bar 50T $\mu \mathrm{m}$ ) in each group were shown. (B) Statistical analysis of integrated density of GFAP in each group $(n=6)$. (C) GFAP expression in spinal cord detected by western blotting after CCI. (D) Statistical analysis of the differences between groups $(\mathrm{n}=6) .{ }^{*} p<0.05$; ${ }^{*} p<$ 0.05 , versus the CCI group.

increased in the vehicle group when compared to the sham group (Fig. 4A, a,b); intrathecal injection of Tacrine attenuated the expression of CGRP in the spinal dorsal horn (Fig. 4A, $\mathrm{b}, \mathrm{c})$. Statistical result of the density of CGRP-positive fibers between groups was shown in Fig. 4A, d. NF200 protein not only reflects the neuronal cytoskeleton but also reflects the functional status after injury. As shown in Fig. 4B, we found that administration of Tacrine increased the expression of NF200 in the superficial layers of spinal cord. The results indicate that Tacrine exerts therapeutic effects on neuropathic pain after CCI. 
A
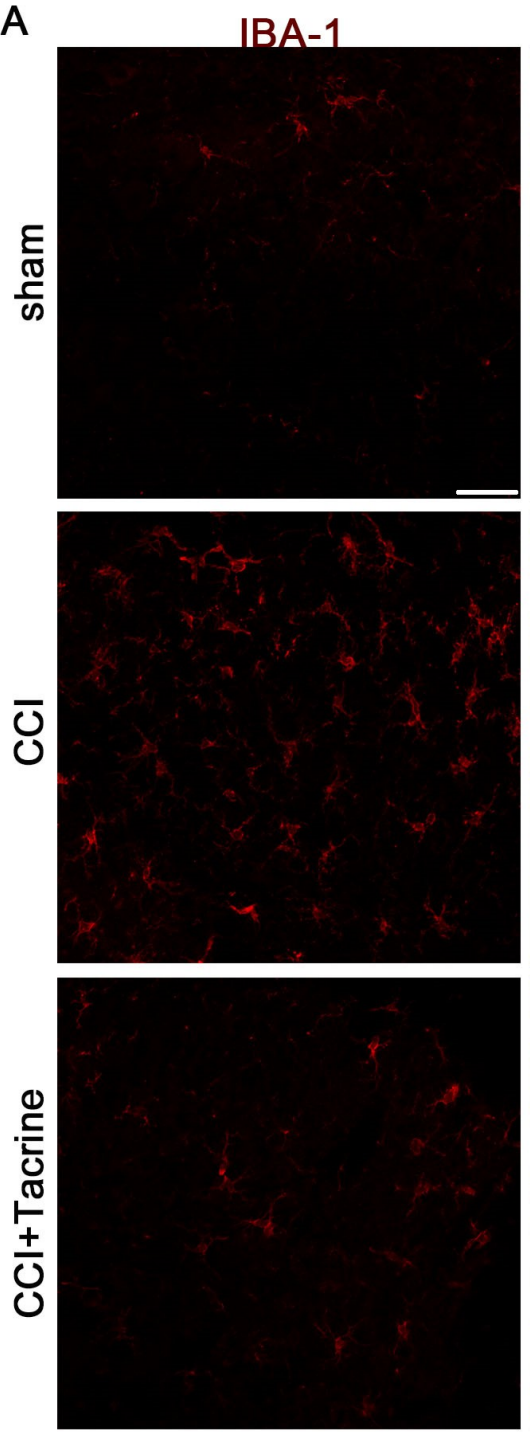

B

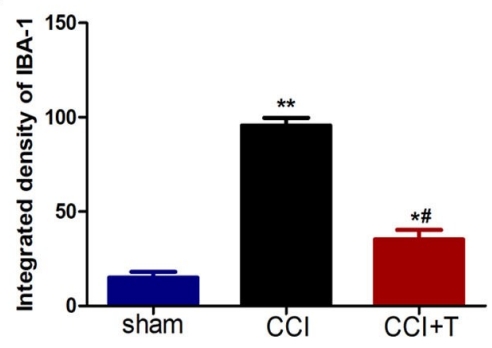

DAPI
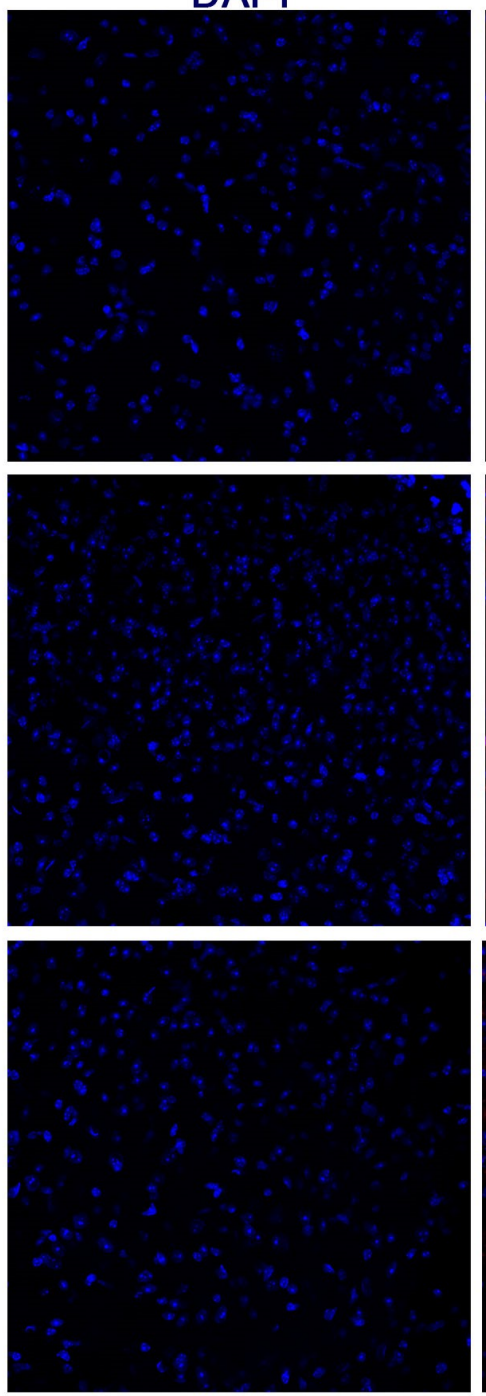

C

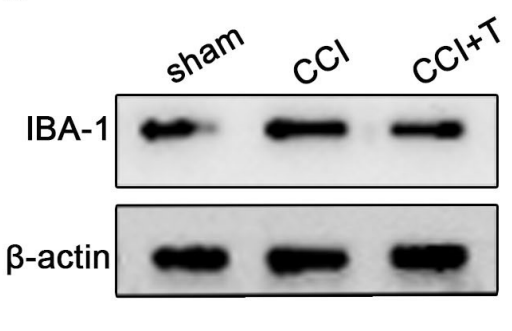

Merge
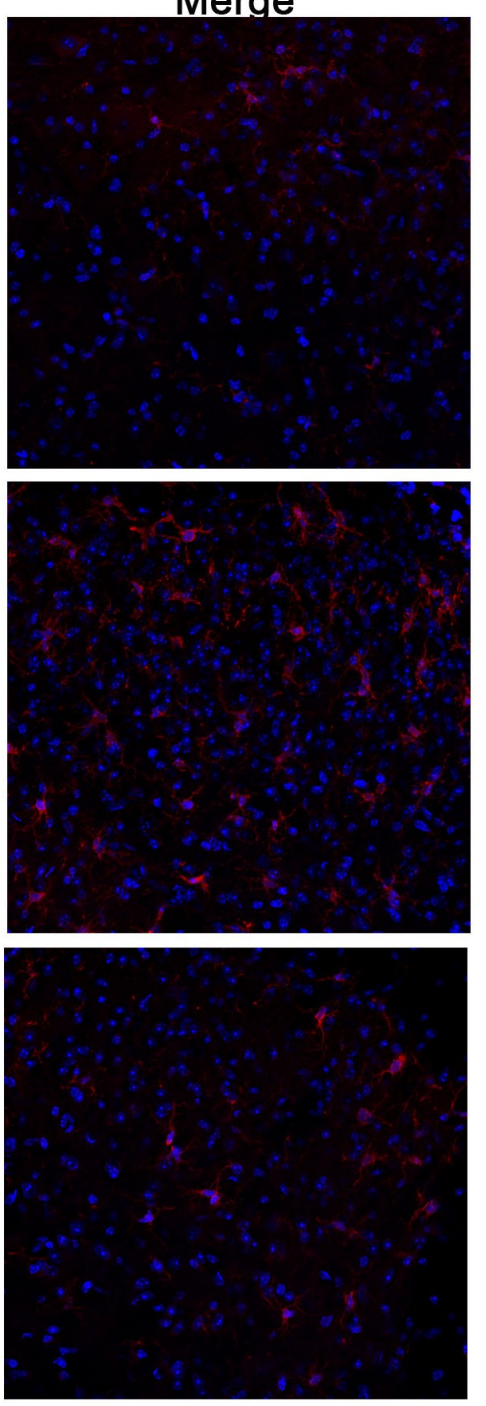

D

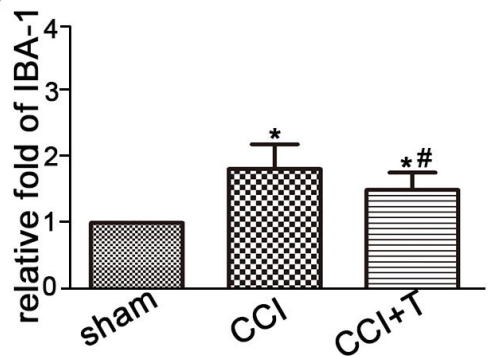

F I G U R E 6. Tacrine decreased the expression of IBA-1 after CCI-injury. (A) Representative immunofluorescence of the microglial marker IBA-1 on the ipsilateral spinal cord (scale bar $50 \mu \mathrm{m}$ ) in each group were shown. (B) Statistical analysis of integrated density of in each group $(n=6)$. (C) IBA-1 expression in spinal cord detected by western blotting after CCI. (D) Statistical analysis of the differences between groups $(\mathrm{n}=6) .{ }^{*} p<0.05,{ }^{* *} p<0.01$ versus the sham group; ${ }^{*} p<0.05$, versus the CCI group.

\subsection{Administration of Tacrine significantly suppressed the activation of astrocytes and microglia induced by CCI}

The activation of glial cells was evaluated by immunofluorescence staining and western blotting. We observed that GFAP detected by immunofluorescence staining was highly expressed after CCI and was decreased after treatment with
Tacrine (Fig. 5A). The statistical result of GFAP in each group was shown in Fig. 5B. The protein content of GFAP in each group was detected by western blotting (Fig. 5C); we detected that the level of GFAP protein increased in CCI mice, and Tacrine decreased the expression of GFAP after CCI from $2.54 \pm 0.19$ to $2.01 \pm 0.02(p<0.05)$ (Fig. 5D). It was also detected that IBA-1, examined by immunofluorescence stain- 

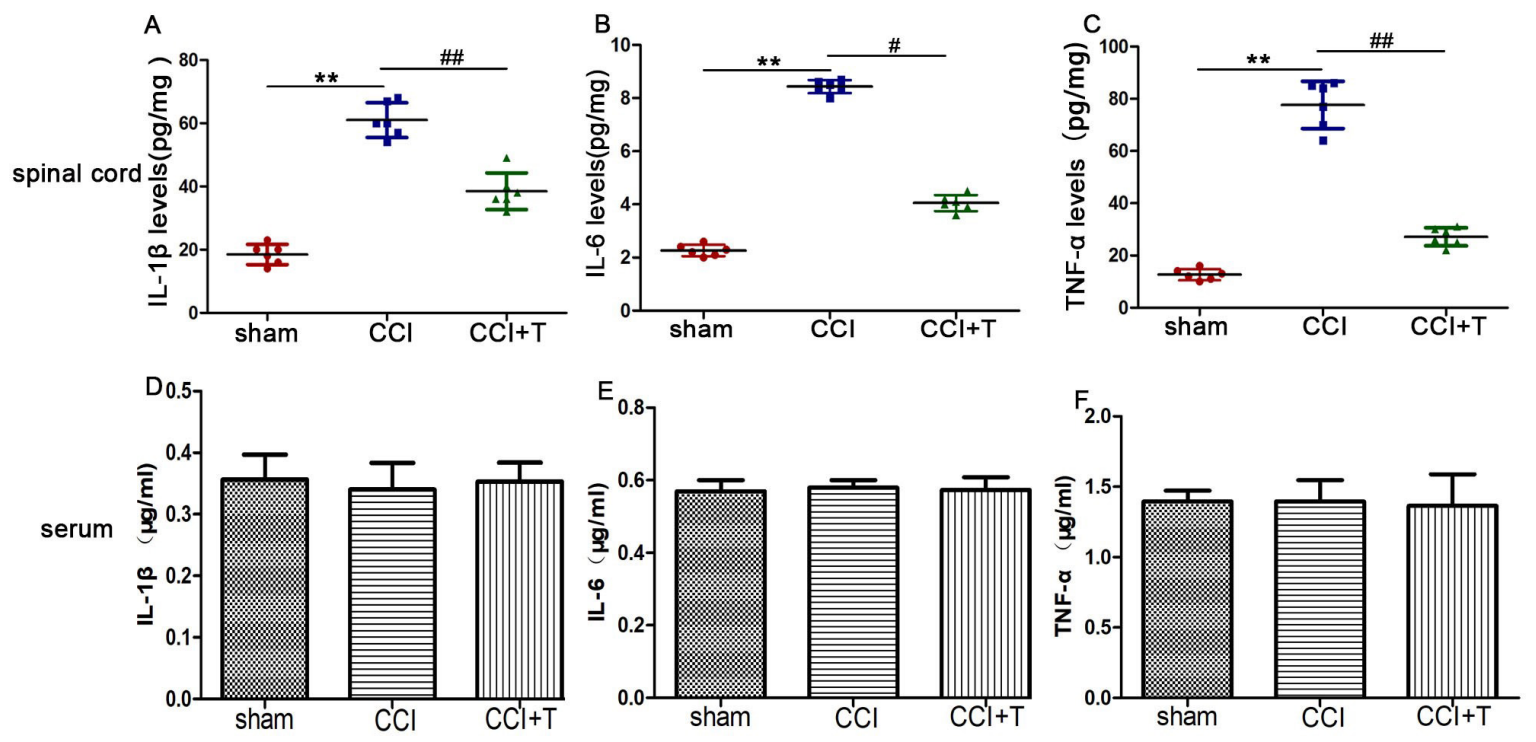

F I G U RE 7. Tacrine decreased the level of IL-1 $\beta$, IL-6 and TNF- $\alpha$ in spinal cord but not in serum. (A) Spinal cord level of IL-1 $\beta$. (B) Spinal cord level of IL-6. (C) Spinal cord level of TNF- $\alpha$. (D) Blood plasma level of IL-1 $\beta$. (E) Blood plasma of IL-6. (F) Blood plasma of TNF- $\alpha$. Values represent mean $\pm \operatorname{SEM}(\mathrm{n}=6)$. ${ }^{*} p<0.05,{ }^{*} p<0.01$ versus the sham group; ${ }^{*} p<$ 0.05 , versus the CCI group.

ing, was obviously increased after CCI but reduced sharply after treatment with Tacrine (Fig. 6B). There was a consistent relationship between IBA-1 and GFAP. Furthermore, IBA-1 protein level was examined by western blotting (Fig. 6C), and was found to decrease from $1.87 \pm 0.13$ in CCI group to 1.52 $\pm 0.096(p<0.05)$ in the Tacrine group (Fig. 6D). The result verified that Tacrine suppressed the activation of glial cells after CCI.

\subsection{Administration of Tacrine significantly reduced the pro-inflammation cytokines in ipsilateral spinal cord but not in serum after CCI}

Glia reaction is an important indicator of neuroinflammation after NP and should be associated with changes of cytokines. To test this, levels of IL-1 $\beta$ (Fig. 7A,D), IL-6 (Fig. 7B,E), and TNF- $\alpha$ (Fig. 7C,F) were examined by ELISA in both ipsilateral spinal cord and serum on day 28 after CCI. NP induced an obvious increase of IL-1 $\beta(p<0.01)$, IL-6 $(p<0.01)$ and TNF- $\alpha(p<0.01)$ in ipsilateral cord but not in serum $(p>$ $0.05)$. CCI may have induced local inflammation but not affected systemic inflammation. After the administration of Tacrine, levels of these pro-inflammatory cytokines in spinal cord significantly decreased. The empirical result shown was that the neuroinflammation induced by CCI was mainly involved in ipsilateral spinal cord, and intrathecal injection of Tacrine significantly alleviated NP possibly by reducing proinflammation cytokines.

\subsection{The administration of Tacrine inhibits the JAK2/STAT3 signaling pathway in CCI-induced NP}

The JAK2/STAT3 signaling pathway is a critical mediator influenced by cytokines in the neuroinflammation [26]. There- fore, inhibition of the JAK2 signaling pathway is an important strategy for alleviating neuropathic pain. To assess whether the administration of Tacrine after CCI imposes any effect on JAK2/STAT3 signaling, we studied JAK2- and STAT3immunoreactive cells by immunofluorescence of spinal sections and detected their protein expression. Only a few neurons (NeuN-positive) were positive for JAK2 in the sham group. However, CCI induced JAK2 expression in a higher number of spinal neurons (Fig. 8A). After intrathecal injection of Tacrine, the JAK2-positive neurons decreased compared with the vehicle group. To further confirm these results, we collected samples and performed western blotting (Fig. 8B). As shown in Fig. $8 \mathrm{C}$, the CCI obviously increased the expression of JAK2 compared with the sham group $(p<0.01)$, whereas the elevation of JAK2 was inhibited by Tacrine ( $p$ $<0.05)$. Similarly, CCI induced the increased expression of STAT3 in spinal neurons (Fig. 8D), and an elevation of STAT3 protein (Fig. 8E), whereas Tacrine resulted in a downregulation of STAT3 including less STAT3-immunoreactive neurons and lower levels of proteins than the CCI group ( $p$ $<0.01$ ) (Fig. 8F). These data suggest that intrathecal injection of Tacrine effectively inhibits the JAK2/STAT3 signaling pathway, which consequently contributes to alleviation of NP.

\section{Discussion}

Previous studies have reported that Tacrine promoted rapid the recovery from spinal cord injury of locomotor activities in both larval and adult zebrafish. Tacrine treatment upregulated the expression of L1 [23]. In the present study, we demonstrated that Tacrine could exert a significant effect on alleviating mechanical allodynia and cold and heat hyperalgesia, and that it also inhibited glial activation and the expression proinflammation cytokines. Tacrine may be involve the recovery progress from neuropathic pain by mimicking the effect of L1. 

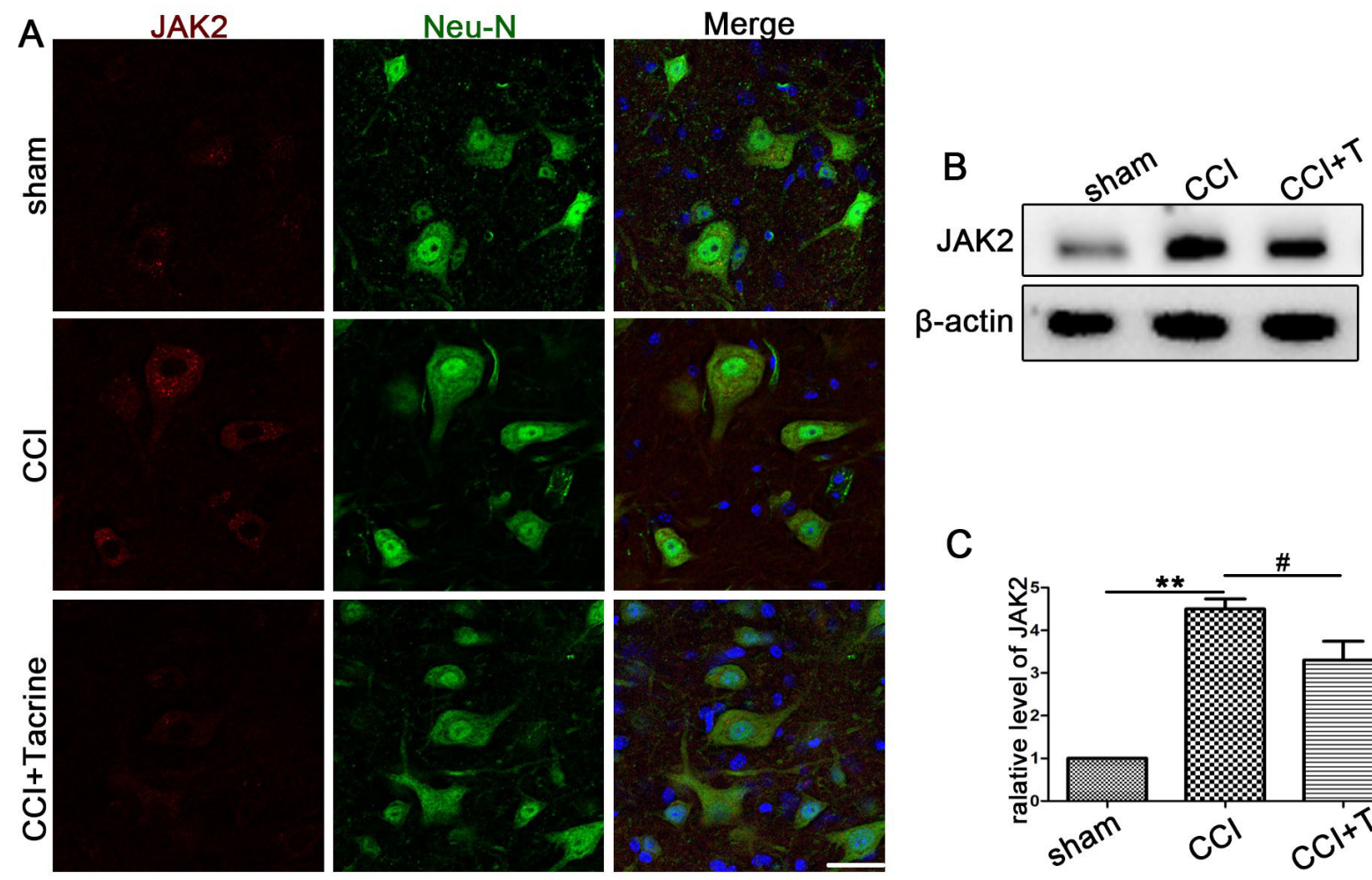

C
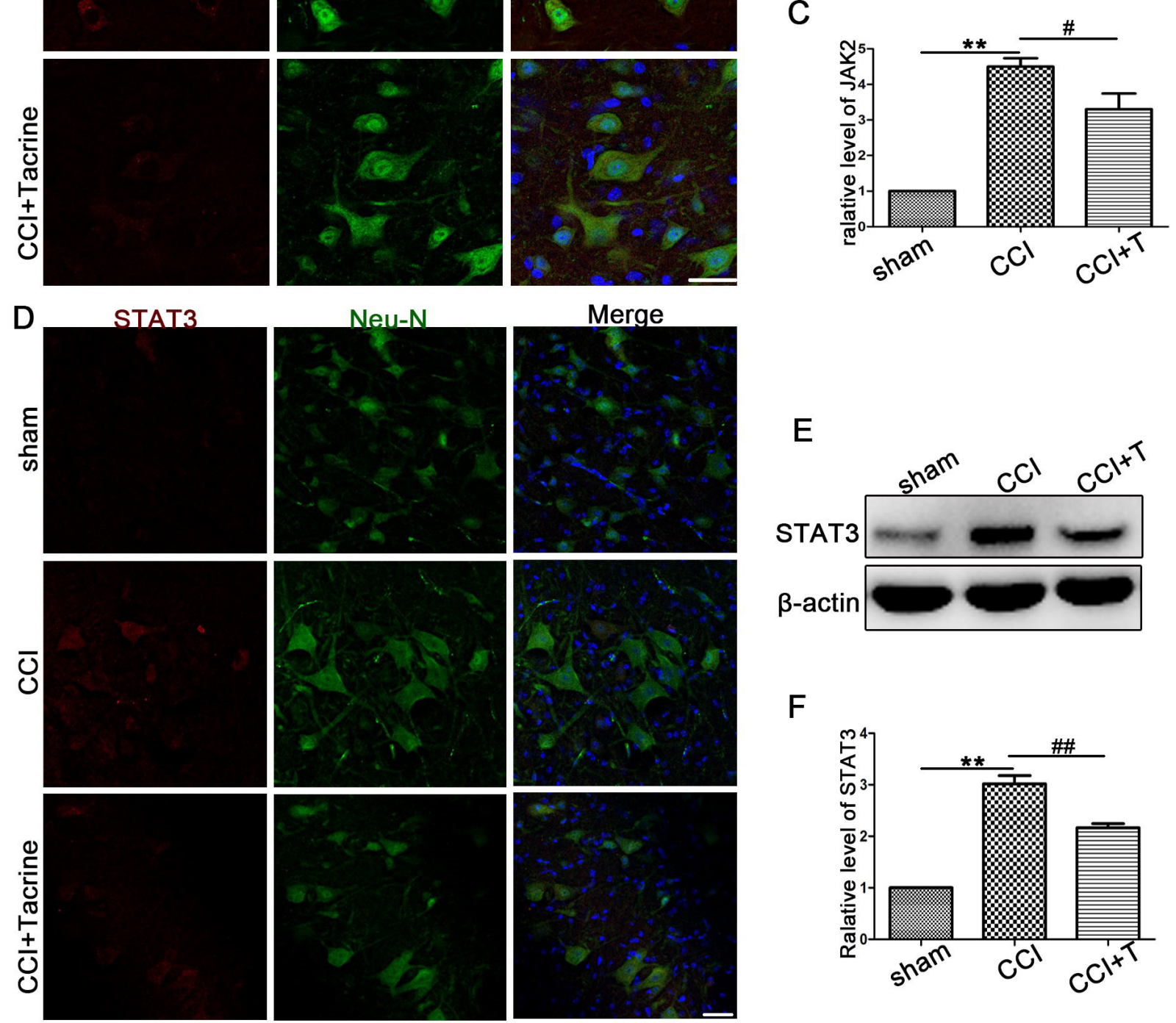

$\mathrm{F}$

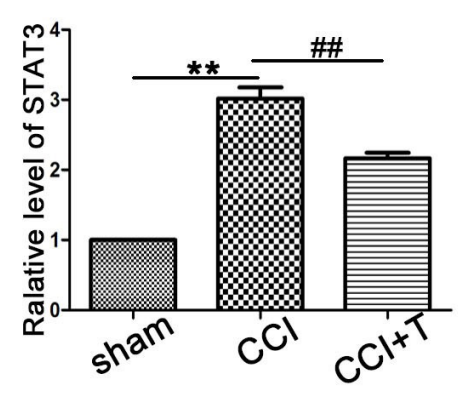

F I G U R E 8. The effect of Tacrine on the expression of JAK2/STAT3 in spinal cord. (A) Representative immunofluorescence staining of JAK2, the neuron marker Neu-N and add the nucleus marker DAPI overlay to form Merge (scale bar $50 \mu \mathrm{m}$ ). (B) JAK2 expression in spinal cord detected by western blotting of each group. (C) Statistical analysis of the protein differences between groups $(n=6)$. (D) Representative immunofluorescence staining of STAT3, the neuron marker Neu-N and add the nucleus marker DAPI overlay to form Merge (scale bar $100 \mu \mathrm{m}$ ). (E) STAT3 expression in spinal cord detected by western blotting of each group. (F) Statistical analysis of the protein differences between groups $(n=6)$.

CGRP, as an important mediator of pain, is distributed widely in both the peripheral and central nervous systems [27]. In mice, CGRP plays a pronociceptive role in response to both mechanical and thermal stimuli [28]. Our data show that CGRP-positive nociceptive fibers became markedly denser in spinal dorsal horn after CCI. Conversely, the density of CGRP- 
positive fibers was significantly lower after the administration of Tacrine. NF200 proteins reflect the morphology and functional status of neurons [29]. These data implied the potential antinociceptive effect of Tacrine in neuropathic pain mice. Moreover, Tacrine might support favorable conditions for functional reconstruction after CCI injury.

This project used the CCI model of neuropathic pain because it involves both components of neuroinflammation with increasing levels of pro-inflammatory cytokines [30]. Hence, spinal cytokine modulation could induce and specifically block mechanisms that prevent behavioral hypersensitivity [31, 32]. Neuroinflammation is primarily characterized by the presence of major cytokines, such as interleukin-1 $\beta$ (IL-1 $\beta$ ), interleukin-6 (IL-6) and tumor necrosis factor- $\alpha$ (TNF- $\alpha$ ) [33]. Other studies have also reported the role of IL-6 in the pathogenesis of NP [34, 35]. Satellite glial cells express IL-6 and corresponding receptors in the rat neuropathic pain model [30]. In our project, IL-1 $\beta$, IL-6 and TNF- $\alpha$ reached maximum levels on day 7 after nerve injury and remained at a high level until day 28 . We detected that the expression of IL- $1 \beta$, IL- 6 and TNF- $\alpha$ in spinal cord was down-regulated after intrathecal Tacrine injection. Furthermore, we presume that Tacrine decreased the expression cytokines by inhibiting the proliferation and activation of glial cells. Regulation of the activation state of glial cells has emerged as a key mechanism in NP, with it being particularly characterized by the synthesis and release of inflammatory cytokines able to modulate synaptic activity and further modulate pain sensitivity [36, 37]. Our findings can be explained by a neuroinflammatory response resulting in the sensitization of nociceptive neurons and neuropathic pain [38, 39].

Recent studies have shown that some anti-inflammation analgesics exert anti-nociception via a signaling cascade response [40]. JAK2, as one of cytoplasmic tyrosine kinases, is associated with cytokine receptors and catalysts of downstream STAT3 activation [41]. The over-activated JAK2/STAT3 signal pathway may then ignite the neuropathic pain cascade by facilitating interaction with cytokines. However, there are few reports on the potential role of L1 agonist on JAK2/STAT3 signaling regulation. In our study, Tacrine treatment reversed the expression of JAK2/STAT3 signal pathway, which had shown a marked increase after CCI surgery. Tacrine might have protective effects via the suppression of cytokines and the blockade of JAK2-mediated activation of STAT3. We thereby inferred that the JAK2/STAT3 signaling pathway is the probable mechanism leading to the protective effects of Tacrine in the mouse CCI model.

In summary, our present study demonstrated the involvement of glial cell over-activation and neuro inflammation in the mouse CCI model. Treatment with Tacrine mitigated pain sensitization, reduced the expression of inflammatory mediators, and inhibited the JAK2/STAT3 pathway after CCI injury.

\section{Conclusions}

Tacrine, which is a small organic compound, is able to mimic the cell adhesion molecule L1. When tacrine treatment was provided throughout the development of neuropathic pain, it exhibited analgesic properties in mechanical allodynia, heat hyperalgesia and cold allodynia. Furthermore, its effect was due to its anti-inflammation property. We demonstrated that Tacrine successfully decreased the level of IL- $1 \beta$, IL- 6 and TNF- $\alpha$ in spinal cord tissues but not in blood plasma. Tacrine also significantly depressed the activation of astrocyte and microcyte in superficial laminae of spinal dorsal horn and inhibited the JAK2/STAT3 signal pathway after peripheral nerve injury. Therefore, Tacrine is a feasible treatment for neuropathic pain. Further investigation is required to investigate the potential clinical applications of the finding.

\section{AUTHOR CONTRIBUTIONS}

HX designed the study, collected and analyzed the data, SX prepare the manuscript for publication and reviewed the draft of the manuscript.

\section{ETHICS APPROVAL AND CONSENT TO PARTICIPATE}

Ethical approval was obtained from the Ethics Committee of Southern Medical University (SMU.No20171215c060105).

\section{ACKNOWLEDGMENT}

I would like to express my gratitude to all those who help me during the writing the manuscript. Thanks to all the peer reviewer for their opinions and suggestions for our study.

\section{FUNDING}

This work was supported by the National Natural Science Foundation of China (Grant No. 81671192 and No. 81771315).

\section{CONFLICT OF INTEREST}

The authors declare no conflict of interest.

\section{REFERENCES}

[1] Torrance N, Ferguson JA, Afolabi E, Bennett MI, Serpell MG, Dunn $\mathrm{KM}$, et al. Neuropathic pain in the community: more under-treated than refractory? Pain. 2013; 154: 690-699.

[2] Sandkühler J. Models and mechanisms of hyperalgesia and allodynia. Physiological Reviews. 2009; 89: 707-758.

[3] Matsuo H, Uchida K, Nakajima H, Guerrero AR, Watanabe S, Takeura $\mathrm{N}$, et al. Early transcutaneous electrical nerve stimulation reduces hyperalgesia and decreases activation of spinal glial cells in mice with neuropathic pain. Pain. 2014; 155: 1888-1901.

[4] Gao Y, Ji R. Chemokines, neuronal-glial interactions, and central processing of neuropathic pain. Pharmacology \& Therapeutics. 2010; 126: 56-68.

[5] Nicolas C, Peineau S, Amici M, Csaba Z, Fafouri A, Javalet C, et al. The JAK/STAT pathway is involved in synaptic plasticity. Neuron. 2012; 73: 374-390.

[6] Singh Jaggi A, Singh N. Therapeutic targets for the management of 
peripheral nerve injury-induced neuropathic pain. CNS \& Neurological Disorders-Drug Targets. 2011; 10: 589-609.

[7] Wood P, Schachner M, Bunge R. Inhibition of Schwann cell myelination in vitro by antibody to the L1 adhesion molecule. Journal of Neuroscience. 1990; 10: 3635-3645.

[8] Hoschouer EL, Yin FQ, Jakeman LB. L1 cell adhesion molecule is essential for the maintenance of hyperalgesia after spinal cord injury. Experimental Neurology. 2009; 216: 22-34.

[9] Yamanaka H, Obata K, Kobayashi K, Dai Y, Fukuoka T, Noguchi K. Alteration of the cell adhesion molecule L1 expression in a specific subset of primary afferent neurons contributes to neuropathic pain. European Journal of Neuroscience. 2007; 25: 1097-1111.

[10] Barbin G, Aigrot MS, Charles P, Foucher A, Grumet M, Schachner M, et al. Axonal cell-adhesion molecule L1 in CNS myelination. Neuron Glia Biology. 2004; 1: 65-72.

[11] Lavdas AA, Papastefanaki F, Thomaidou D, Matsas R. Cell adhesion molecules in gene and cell therapy approaches for nervous system repair. Current Gene Therapy. 2011; 11: 90-100.

[12] Irintchev A, Schachner M. The injured and regenerating nervous system: immunoglobulin superfamily members as key players. Neuroscientist. 2012; 18: 452-466.

[13] Skaper SD. Neuronal growth-promoting and inhibitory cues in neuroprotection and neuroregeneration. Methods in Molecular Biology. 2012; 846: $13-22$.

[14] Kamiguchi H. The mechanism of axon growth: what we have learned from the cell adhesion molecule L1. Molecular Neurobiology. 2003; 28 : 219-228.

[15] Maness PF, Schachner M. Neural recognition molecules of the immunoglobulin superfamily: signaling transducers of axon guidance and neuronal migration. Nature Neuroscience. 2007; 10: 19-26.

[16] Doherty P, Williams G, Williams EJ. CAMs and axonal growth: a critical evaluation of the role of calcium and the MAPK cascade. Molecular and Cellular Neurosciences. 2000; 16: 283-295.

[17] Loers G, Chen S, Grumet M, Schachner M. Signal transduction pathways implicated in neural recognition molecule L1 triggered neuroprotection and neuritogenesis. Journal of Neurochemistry. 2005; 92: 1463-1476.

[18] Kataria H, Lutz D, Chaudhary H, Schachner M, Loers G. Small molecule agonists of cell adhesion molecule L1 mimic L1 functions in vivo. Molecular Neurobiology. 2016; 53: 4461-4483.

[19] Binder A, Baron R. The pharmacological therapy of chronic neuropathic pain. Deutsches Arzteblatt International. 2016; 113: 616-625.

[20] Nishikawa N, Nomoto M. Management of neuropathic pain. Journal of General and Family Medicine. 2017; 18: 56-60.

[21] Knapp MJ, Knopman DS, Solomon PR, Pendlebury WW, Davis CS, Gracon SI. A 30-week randomized controlled trial of high-dose tacrine in patients with Alzheimer's disease. The Tacrine Study Group. The Journal of the American Medical Association. 1994; 271: 985-991.

[22] Lin H, Li Q, Gu K, Zhu J, Jiang X, Chen Y, et al. Therapeutic agents in Alzheimer's disease through a multi-targetdirected ligands strategy: recent progress based on tacrine core. Current Topics in Medicinal Chemistry. 2017; 17: 3000-3016.

[23] Sahu S, Zhang Z, Li R, Hu J, Shen H, Loers G, et al. A small organic compound mimicking the $\mathrm{L} 1$ cell adhesion molecule promotes functional recovery after spinal cord injury in zebrafish. Molecular Neurobiology. 2018; 55: 859-878.

[24] Yi Y, Cai L, Shao Y, Xu M, Yi J. The protective role of tacrine and donepezil in the retina of acetylcholinesterase knockout mice. International Journal of Ophthalmology. 2015; 8: 884-890.

[25] Nadal X, Baños J, Kieffer BL, Maldonado R. Neuropathic pain is enhanced in delta-opioid receptor knockout mice. European Journal of Neuroscience. 2006; 23: 830-834.

[26] Xue Z, Shen L, Wang Z, Hui S, Huang Y, Ma C. STAT3 inhibitor
WP1066 as a novel therapeutic agent for bCCI neuropathic pain rats. Brain Research. 2014; 1583: 79-88.

[27] Lin Y, Liu H, Day Y, Chang C, Hsu P, Chen J. Activation of NPFFR2 leads to hyperalgesia through the spinal inflammatory mediator CGRP in mice. Experimental Neurology. 2017; 291: 62-73.

[28] Malon JT, Cao L. Calcitonin gene-related peptide contributes to peripheral nerve injury-induced mechanical hypersensitivity through CCL5 and p38 pathways. Journal of Neuroimmunology. 2016; 297: 6875

[29] Hulme CH, Brown SJ, Fuller HR, Riddell J, Osman A, Chowdhury J, et al. The developing landscape of diagnostic and prognostic biomarkers for spinal cord injury in cerebrospinal fluid and blood. Spinal Cord. 2017; 55: 114-125.

[30] Farghaly HSM, Mahmoud AM, Abdel-Sater KA. Effect of dexmedetomidine and cold stress in a rat model of neuropathic pain: role of interleukin6 and tumor necrosis factor- $\alpha$. European Journal of Pharmacology. 2016; 776: 139-145.

[31] Sacerdote P, Franchi S, Moretti S, Castelli M, Procacci P, Magnaghi $\mathrm{V}$, et al. Cytokine modulation is necessary for efficacious treatment of experimental neuropathic pain. Journal of Neuroimmune Pharmacology. 2013; 8: 202-211.

[32] Whitehead KJ, Smith CGS, Delaney S, Curnow SJ, Salmon M, Hughes $\mathrm{JP}$, et al. Dynamic regulation of spinal pro-inflammatory cytokine release in the rat in vivo following peripheral nerve injury. Brain, Behavior, and Immunity. 2010; 24: 569-576.

[33] Mori T, Miyamoto T, Yoshida H, Asakawa M, Kawasumi M, Kobayashi T, et al. IL- $1 \beta$ and TNF $\alpha$-initiated IL-6-STAT3 pathway is critical in mediating inflammatory cytokines and RANKL expression in inflammatory arthritis. International Immunology. 2011; 23: 701-712.

[34] Zhou Y, Liu Z, Liu Z, Chen S, Li M, Shahveranov A, et al. Interleukin-6: an emerging regulator of pathological pain. Journal of Neuroinflammation. 2016; 13: 141.

[35] Wei X, Na X, Liao G, Chen Q, Cui Y, Chen F, et al. The up-regulation of IL-6 in DRG and spinal dorsal horn contributes to neuropathic pain following L5 ventral root transection. Experimental Neurology. 2013; 241: $159-168$

[36] Ji R, Berta T, Nedergaard M. Glia and pain: is chronic pain a gliopathy? Pain. 2013; 154: S10-S28.

[37] Kronschläger MT, Drdla-Schutting R, Gassner M, Honsek SD, Teuchmann HL, Sandkühler J. Gliogenic LTP spreads widely in nociceptive pathways. Science. 2016; 354: 1144-1148.

[38] Zhu X, Cao S, Zhu M, Liu J, Chen J, Gao Y. Contribution of chemokine CCL2/CCR2 signaling in the dorsal root ganglion and spinal cord to the maintenance of neuropathic pain in a rat model of lumbar disc herniation. Journal of Pain. 2014; 15: 516-526.

[39] Komirishetty P, Areti A, Sistla R, Kumar A. Morin mitigates chronic constriction injury (CCI)-induced peripheral neuropathy by inhibiting oxidative stress induced PARP over-activation and neuroinflammation. Neurochemical Research. 2016; 41: 2029-2042.

[40] Busch-Dienstfertig M, Labuz D, Wolfram T, Vogel NN, Stein C. JAKSTAT1/3-induced expression of signal sequence-encoding proopiomelanocortin mRNA in lymphocytes reduces inflammatory pain in rats. Molecular Pain. 2012; 8: 83.

[41] Murray PJ. The JAK-STAT signaling pathway: input and output integration. Journal of Immunology. 2007; 178: 2623-2629.

How to cite this article: Hua-Li $\mathrm{Xu}$, Shi-Yuan $\mathrm{Xu}$. Tacrine alleviates neuropathic pain in mice by mimicking the cell adhesion molecule L1. Signa Vitae. 2021;17(4):140-150. doi: $10.22514 /$ sv.2021.081. 